\title{
The Planck and LHC results and particle physics
}

\section{Fedor BEZRUKOV*}

Physics Department, University of Connecticut, 2152 Hillside Road, Storrs, CT 06269-3046, U.S.A.

RIKEN-BNL Research Center, Brookhaven National Laboratory,

Bldg. 510a, Upton, NY 11973-5000, U.S.A.

E-mail: Fedor.Bezrukovauconn.edu

I will discuss the recent LHC and Planck results, which are completely compatible with the Standard Model of particle physics, and the standard cosmological model $(\Lambda \mathrm{CDM})$, respectively. It turns out that the extension of the Standard Model is, of course, required, but can be very minimal. I will discuss also what future measurements may be important to test this approach.

The European Physical Society Conference on High Energy Physics 18-24 July, 2013

Stockholm, Sweden

${ }^{*}$ Speaker. 


\section{Introduction and overview of current experimental results}

Current results from LHC and Planck show incredible agreement with the Standard Model of particle physics (SM) on the one hand and standard cosmological model ( $\Lambda \mathrm{CDM})$ on the other hand. Moreover, in cosmology the Planck results [1] constrain the primordial perturbations to have low amplitude of tensor modes and have no significant non-Gaussianities, thus favoring single field inflation with relatively low inflationary scale. In particle physics a Higgs boson with the mass around $126 \mathrm{GeV}$ was discovered [2,3], and further searches reveal no new particle states and no deviations from the SM in predictions of rare decays.

At the same time extension of the SM is necessary to explain experimental problems, that are neutrino oscillations, baryon asymmetry of the Universe, Dark Matter, and inflation. ${ }^{1}$ Usual extensions of the SM introduce some particle physics at scales far above electroweak scale. In such quantum field theories large (proportional to the mass of heavy particles) radiative contributions to the mass of the Higgs boson emerge, which is one way to see the hierarchy problem. These contributions may be cancelled in e.g. supersymmetric theories, but until now no evidence of supersymmetric partners for SM particles (or other deviations form SM) have been observed.

An alternative solution to the hierarchy problem may be searched in models without new particle physics above electroweak scale. In this case the only heavy scale in the theory is related to the Planck scale defining the quantum gravitational effects, where the problem of quadratic divergences may be different from the ordinary field theory (see [4] for a toy model example). To attempt this approach all the experimental facts mentioned in the previous paragraph should be explained in a theory without heavy particles, see talk by M. Shaposhnikov [5]. The solution to the Dark Matter and baryogenesis can be achieved within the $v$ MSM extension of the SM by three light sterile neutrinos, see talks by M. Shaposhnikov and O. Ruchayskiy, and ref. [6] for review.

This talk gives a short overview of selected simple inflationary models that can be used in such a framework. One way is to add a new (light) scalar inflaton and make it consistent with observations by a small non-minimal coupling, which is a rather conservative particle physics model of inflation. Second way is to use large non-minimal coupling for the Higgs boson itself with the advantage of having no additional degrees of freedom, but with potentially complicated quantum dynamics at high energies. The third method is the $R^{2}$ inflation, which provides the solution completely within the gravitational part of the theory.

\section{Simple inflationary models}

\subsection{Light non-minimally coupled inflaton with quartic potential}

Probably the simplest and most widely known inflationary model uses an additional dedicated scalar field [7]. The proper normalization of the density perturbations requires the potential of the field to be very flat. In case of the quartic potential this defines the self coupling constant $\beta \sim 1.5 \times$ $10^{-13}$. However, the energy scale during inflation turns out to be not small enough and significant amount of tensor perturbations is generated. The recent Planck results exclude this model at more than $95 \%$ confidence level. This does not mean that models with simple quartic potentials should

\footnotetext{
${ }^{1}$ Strictly speaking, one should explain the observed nearly scale invariant spectrum of primordial perturbations.
} 
be abandoned as inflationary theories. For a scalar field an additional non-minimal coupling to gravity can be added to the model $[8,9]$. Let us note that such a coupling is even required by the renormalization of a scalar field in a curved space-time background.

The part of the action responsible for inflation is then

$$
S_{\mathrm{NM} X^{4}}=\int \sqrt{-g} d^{4} x\left(-\frac{M_{P}^{2}+\xi X^{2}}{2} R+\frac{1}{2} \partial_{\mu} X \partial^{\mu} X-\frac{\beta}{4} X^{4}\right)
$$

where we neglected the possibility of $X$ having a nonzero vacuum expectation value contributing significantly to the Planck mass (see [10] for further discussion). The simplest way to analyze this model is to make the conformal transformation of the metric

$$
g_{\mu v} \rightarrow \tilde{g}_{\mu v}=\Omega^{2} g_{\mu v}, \quad \Omega^{2}=1+\xi X^{2} / M_{P}^{2}
$$

and redefine the inflaton field $X \rightarrow \chi$ to regain canonically normalized kinetic term. In the new variables $\tilde{g}_{\mu v}$ and $\chi$ (Einstein frame, as opposed to the original Jordan frame) gravity couples minimally, while the inflaton potential gets rescaled according to

$$
U(\chi)=\frac{\beta X^{4}(\chi)}{4 \Omega^{4}(\chi)}, \quad \frac{d \chi}{d X}=\sqrt{\frac{\Omega^{2}+6 \xi^{2} X^{2} / M_{P}^{2}}{\Omega^{4}}} .
$$

This potential is evidently more flat and gives less tensor perturbations.

Starting from this point the analysis is a straightforward slow roll inflation with one field. It turns out that a very well analytic approximation can be achieved by using the following relation for the field value $X_{N}$ at the $N$ e-foldings $(1+6 \xi) X_{N}^{2} / M_{P}^{2}=8(N+1)$. Though it is formally correct only at small $\xi$, it smoothly interpolates between the small $\xi$ and large $\xi$ regimes (for large $N$ ) [11]. The resulting slow roll parameters can be obtained now by usual means [11, 8, 9], and interpolate between zero $\xi$ and large $\xi$ limits (see fig. 1). In particular, the tensor-to-scalar ratio $r$ drops rapidly with increasing $\xi$ and becomes compatible with observations for $\xi \gtrsim 0.001$. The self coupling constant $\beta$ is determined by the $\mathrm{CMB}$ normalization and interpolates between the zero $\xi$ value and the large $\xi$ case $\beta \simeq(47000 / \xi)^{2}$.

The number of e-foldings is not in fact arbitrary for quartic inflation, because after the end of the slow roll the evolution of the Universe is dominated by the oscillations of the field in quartic potential which corresponds to the radiation dominated expansion. Thus, though the real reheating (transfer of the energy to the SM degrees of freedom) is not be immediate, for the purposes of the estimate of $N$ for the pivot scale $k / a=0.002 \mathrm{Mpc}^{-1}$ the "reheating" is immediate, leading to $N \simeq 60$, see [11].

Note that non-minimal coupling lessens the concerns that may appear in the large field inflation because of the field spanning superplanckian range during inflation. For the minimally coupled inflation the field changes by about $X_{N} \sim 22 M_{P}$, while for $\xi \sim 1$ only by $8 M_{P}$. For $\xi>1$ the situation becomes more complicated because of the additional scales $M_{P} / \xi$ and $M_{P} / \sqrt{\xi}$ appearing in the model.

To complete the model one should couple the inflationary sector to the SM. Here we would like to make an additional assumption that the scale invariance is only broken in the inflaton sector. Then the scalar part of the model Lagrangian density becomes

$$
\mathscr{L}=\frac{1}{2} m_{X}^{2} X^{2}-\frac{\beta}{4} X^{4}-\lambda\left(H^{\dagger} H-\frac{\alpha}{\lambda} X^{2}\right)^{2}
$$


where $H$ is the Higgs field doublet and $\alpha$ is the only constant controlling the interaction between the SM and inflationary sector. The only free parameter here is $\alpha$ ( $\lambda$ is determined by the Higgs boson mass, and $\beta$ by the CMB normalization). From the requirement of good cosmological evolution $\alpha$ can not be too large not to spoil the inflationary potential with radiative corrections; it can not be too small in order to get sufficient reheating temperature (here we mean transfer of energy into the SM degrees of freedom) to allow for transfer of the asymmetry from the lepton to the baryon sector by the sphaleron transitions [12]. The resulting bounds on $\alpha$ are $0.7 \times 10^{-11} \lesssim$ $\alpha \lesssim \sqrt{0.1 \times \beta}$, see [13]. For the potential (2.2) these range translates into the bounds on the mass of the $X$ particle $[14,11]$. The resulting inflaton has the mass around $\mathrm{GeV}$ and can be searched in rare decay experiments on $\mathrm{LHCb}$.

Another consequence of (2.2) is that the inflation proceeds along the line $H^{\dagger} H=\frac{\alpha}{\lambda} X^{2}$ in the field space. Thus, the value of the Higgs field $H$ is actually rather large at inflation, of the order $\sim$ $\sqrt{\alpha / \lambda} M_{P}$. This implies the lower bound on the Higgs boson mass similar (but just slightly weaker) than that of the requirement of the absolute stability of the electroweak vacuum (or, equivalently, viability of the Higgs inflation) $[15,16,17]$.

\subsection{Higgs inflation}

For a large value of $\xi$ the the self-coupling constant can be made arbitrarily large. So, instead of adding a new field to the model it is possible to just add the non-minimal coupling to gravity of the Higgs field itself [18]

$$
S_{\mathrm{NMHiggs}}=\int \sqrt{-g} d^{4} x\left(-\xi_{h} H^{\dagger} H R\right) .
$$

The HI case corresponds to the large $\xi_{h} \gg 1$ and negligible vacuum contribution of the non-minimal coupling to the Planck mass, $\xi_{h} v^{2} \ll M_{P}^{2}$. In the unitary gauge $H=\frac{1}{\sqrt{2}}\left(\begin{array}{c}0 \\ v+h\end{array}\right)$ the inflationary analysis is analogous to the previous section with the obvious substitution $X \rightarrow h$ and $\beta \rightarrow \lambda$. For the large Higgs background $h, \chi \gg M_{P} / \xi_{h}$ (relevant for inflation) the potential is

$$
U(\chi) \simeq \frac{\lambda M_{P}^{4}}{4 \xi_{h}^{2}}\left(1-\mathrm{e}^{-\frac{2 \chi}{\sqrt{6} M_{P}}}\right)^{2} .
$$

For small field $h, \chi \ll M_{P} / \xi_{h}$, the model returns to the usual SM regime.

The CMB normalization requirement fixes the non-minimal coupling $\xi_{h} \simeq 47000 \sqrt{\lambda}$, where $\lambda$ is the Higgs boson self-coupling constant taken at the inflationary scale. To the lowest order in $1 / \xi$, the spectral index and the tensor-to-scalar perturbation ratio are $n_{s} \simeq 1-8(4 N+9) /(4 N+3)^{2} \simeq$ $0.967, r \simeq 192 /(4 N+3)^{2} \simeq 0.0031$. The inflation model in the Einstein frame is a simple one field slow-rolling inflation, with all extra degrees of freedom much heavier than the Hubble scale $\left(m \sim M_{P} / \sqrt{\xi_{h}} \gg H \sim M_{P} / \xi_{h}\right)$, so it does not predict any significant non-Gaussianities in the spectrum, and the values of the parameters are well in agreement with observations, see fig. 1 .

Note that reheating is quite effective in this model [19, 20], with $T_{r} \sim 0.3-1.1 \times 10^{14} \mathrm{GeV}$. Precise estimate is complicated, but in any case for $h<M_{P} / \xi$ the expansion of the Universe is governed by the quartic potential of SM and is radiatively dominated, so effectively $T_{r} \gtrsim 10^{13} \mathrm{GeV}$ [10]. This reheating temperature corresponds to the number of e-foldings $N \simeq 57.5 \pm 0.2$.

The calculation of the quantum corrections in the Higgs inflation requires additional assumptions on the UV properties of the theory. The variable change (2.1) leads to higher dimensional 

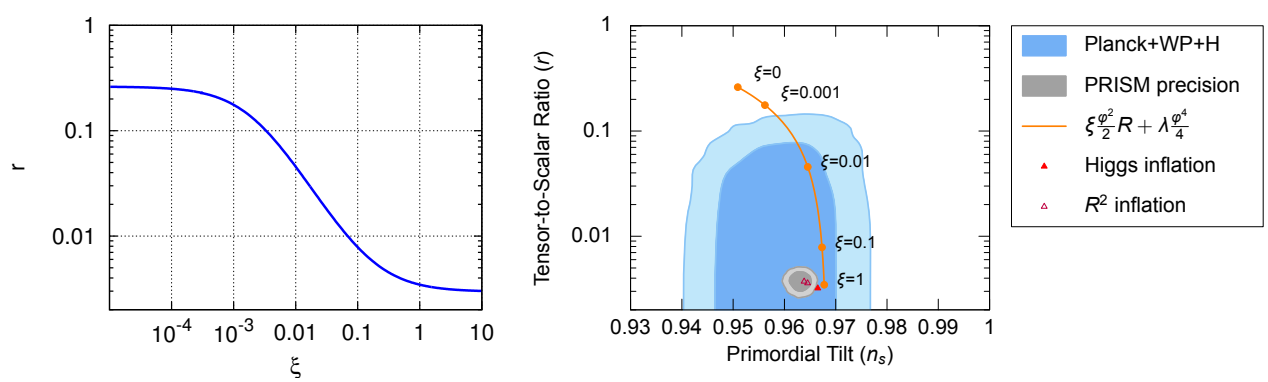

Figure 1: Left: dependence of the tensor-to-scalar ration $r$ on non-minimal coupling constant $\xi$ for the quartic potential. Right: predictions of the described inflationary models for tensor-to-scalar ratio $r$ and spectral index $n_{s}$ and existing experimental bounds from Planck 2012 release [1]. Also shown expected precision from the PRISM experiment [21].

operators in the potential suppressed by the scale $M_{P} / \xi$ leading to tree unitarity violation in electroweak vacuum at this energy. This scale is just slightly above to the Hubble scale at inflation $H \sim \sqrt{\lambda} M_{P} / \xi$. The situation is improved by noting that at inflation the background is very different from the vacuum solution and the small perturbations on top of this background can be safely analyzed at tree level up to a much higher energy (in the Einstein frame up to $M_{P}$ for the gravitational perturbations, and $M_{P} / \sqrt{\xi}$ for SM particle like excitations, see [10, 22]). The inflation starts from the large value of the Higgs field the Higgs inflation can proceed only if the electroweak vacuum is absolutely stable, providing a lower bound on the Higgs mass [15].

\section{$2.3 R^{2}$ inflation}

A related mechanism of inflation emerges in modification of the gravity action alone [23, 24]

$$
S_{R^{2}}=\int \sqrt{-g} d^{4} x\left(-\frac{M_{P}^{2}}{2} R+\frac{M_{P}^{2}}{6 \mu^{2}} R^{2}\right) .
$$

As far as this is a theory with higher order derivatives it has an additional degree of freedom, scalaron $\phi(x)$, which emerges in the simplest way in the conformally transformed (Einstein) frame

$$
g_{\mu v} \rightarrow \tilde{g}_{\mu v}=\mathrm{e}^{\sqrt{2 / 3} \phi / M_{p}} g_{\mu v},
$$

with the Einstein frame action having exactly the form

$$
S_{E F}=\int \sqrt{-\tilde{g}} d^{4} x\left\{-\frac{M_{P}^{2}}{2} \tilde{R}+\frac{1}{2} \partial_{\mu} \phi \partial^{\mu} \phi-\frac{3 \mu^{2} M_{P}^{2}}{4}\left(1-\mathrm{e}^{-\frac{2 \phi}{\sqrt{6} M_{P}}}\right)^{2}\right\} .
$$

In the inflationary domain this potential coincides with that of the Higgs inflation (2.3), so the inflationary predictions of these models are very similar. However, the reheating process here proceeds via Planck scale suppressed operators $[24,25]$ leading to significantly lower reheating temperature $T_{r} \sim 3.1 \times 10^{9} \mathrm{GeV}$ (or even lower for conformally coupled Higgs boson [25]). This leads to a different number of e-foldings $N \simeq 54[26,25]$ and slightly modified CMB predictions, see fig. 1. The next generation CMB experiments may be able to reach the required precision.

Another important feature of the $R^{2}$ inflation is that it does not require the Higgs field to reach large values throughout the evolution of the Universe, thus making it possible to work even if the 
electroweak vacuum is metastable [26, 25]. Also, because the potential in (2.4) is exact the cut-off scale in $R^{2}$ inflation is just $M_{P}$, what is immediately seen after expanding the potential in power series. Note, that this effect is similar to what happens in the inflationary models with non-minimal coupling in the induced gravity regime. (in particular the perturbative UV-completion of the Higgs inflation [27]).

\section{Conclusions}

I described here three inflationary models which can be realized on top of the SM without introduction of heavy particle states in the theory. This is important if one wants to evade quadratic divergences associated with heavy particles in the theory, and may allow to connect inflationary physics with low energy phenomenology.

The main way to distinguish these models is the improved measurements of the parameters of the primordial perturbations, that is the spectral index and tensor-to-scalar ratio. The not so small tensor-to-scalar ratio can easily distinguish the non-minimally coupled quartic inflation, and possibly even determine the value of the non-minimal coupling $\xi$. Significant improvement in $n_{s}$ measurement may distinguish $R^{2}$ and Higgs inflation.

Another significant measurement is related to the analysis of the stability of the electroweak vacuum. Specifically, the Higgs inflation and light non-minimally coupled inflation described in this talk are possible only if the electroweak vacuum is absolutely stable, while the $R^{2}$ inflation may work even for a metastable one. The current Higgs boson mass can be compatible within experimental and theoretical errors with both stable and metastable electroweak vacuum [15, 28]. The most significant improvement here may be measurement of the top quark mass (or directly the top quark Yukawa constant), strong gauge coupling and, finally, Higgs boson mass itself.

\section{References}

[1] Planck Collaboration, P. Ade et al., Planck 2013 results. I. Overview of products and scientific results, arXiv:1303.5062.

[2] CMS Collaboration, S. Chatrchyan et al., Observation of a new boson at a mass of $125 \mathrm{GeV}$ with the CMS experiment at the LHC, Phys.Lett. B716 (2012) 30-61, [arXiv:1207. 7235].

[3] ATLAS Collaboration, G. Aad et al., Observation of a new particle in the search for the Standard Model Higgs boson with the ATLAS detector at the LHC, Phys.Lett. $\mathbf{B 7 1 6}$ (2012) 1-29, [arXiv:1207.7214].

[4] S. Dubovsky, V. Gorbenko, and M. Mirbabayi, Natural tuning: Towards a proof of concept, JHEP 1309 (2013) 045, [arXiv:1305.6939].

[5] M. Shaposhnikov, Cosmology: theory, arXiv:1311. 4979.

[6] A. Boyarsky, O. Ruchayskiy, and M. Shaposhnikov, The role of sterile neutrinos in cosmology and astrophysics, Ann.Rev.Nucl.Part.Sci. 59 (2009) 191-214, [arXiv:0 901.0011$].$

[7] A. D. Linde, A new inflationary universe scenario: A possible solution of the horizon, flatness, homogeneity, isotropy and primordial monopole problems, Phys. Lett. B108 (1982) 389-393.

[8] D. S. Salopek, J. R. Bond, and J. M. Bardeen, Designing density fluctuation spectra in inflation, Phys. Rev. D40 (1989) 1753. 
[9] D. I. Kaiser, Primordial spectral indices from generalized einstein theories, Phys. Rev. D52 (1995) 4295-4306, [astro-ph/9408044].

[10] F. Bezrukov, The Higgs field as an inflaton, Class.Quant.Grav. 30 (2013) 214001, [arXiv:1307.0708].

[11] F. Bezrukov and D. Gorbunov, Light inflaton after LHC8 and WMAP9 results, JHEP 1307 (2013) 140, [arXiv:1303.4395].

[12] Y. Burnier, M. Laine, and M. Shaposhnikov, Baryon and lepton number violation rates across the electroweak crossover, JCAP 0602 (2006) 007, [hep-ph / 0511246 ].

[13] A. Anisimov, Y. Bartocci, and F. L. Bezrukov, Inflaton mass in the vMSM inflation, Phys. Lett. B671 (2009) 211-215, [arXiv:0809.1097].

[14] F. Bezrukov and D. Gorbunov, Light inflaton hunter's guide, JHEP 1005 (2010) 010, [arXiv:0912.0390].

[15] F. Bezrukov, M. Y. Kalmykov, B. A. Kniehl, and M. Shaposhnikov, Higgs Boson Mass and New Physics, JHEP 1210 (2012) 140, [arXiv: 1205.2893$].$

[16] G. Degrassi, S. Di Vita, J. Elias-Miro, J. R. Espinosa, G. F. Giudice, G. Isidori, and A. Strumia, Higgs mass and vacuum stability in the Standard Model at NNLO, JHEP 1208 (2012) 098, [arXiv:1205.6497].

[17] S. Alekhin, A. Djouadi, and S. Moch, The top quark and Higgs boson masses and the stability of the electroweak vacuum, Phys.Lett. B716 (2012) 214-219, [arXiv: 1207 . 0980].

[18] F. L. Bezrukov and M. Shaposhnikov, The Standard Model Higgs boson as the inflaton, Phys. Lett. B659 (2008) 703-706, [arXiv: 0710.3755$].$

[19] F. Bezrukov, D. Gorbunov, and M. Shaposhnikov, On initial conditions for the Hot Big Bang, JCAP 0906 (2009) 029, [arXiv:0 812.3622 ].

[20] J. Garcia-Bellido, D. G. Figueroa, and J. Rubio, Preheating in the Standard Model with the Higgs-Inflaton coupled to gravity, Phys. Rev. D79 (2009) 063531, [arXiv : 0812 . 462 4].

[21] PRISM Collaboration, P. Andre et al., PRISM (Polarized Radiation Imaging and Spectroscopy Mission): A white paper on the ultimate polarimetric spectro-imaging of the microwave and far-infrared sky, arXiv:1306.2259.

[22] F. Bezrukov, A. Magnin, M. Shaposhnikov, and S. Sibiryakov, Higgs inflation: consistency and generalisations, JHEP 1101 (2011) 016, [arXiv: 1008 .5157].

[23] A. A. Starobinsky, A new type of isotropic cosmological models without singularity, Phys. Lett. $\mathbf{B 9 1}$ (1980) 99-102.

[24] D. Gorbunov and A. Panin, Scalaron the mighty: producing dark matter and baryon asymmetry at reheating, Phys.Lett. B700 (2011) 157-162, [arXiv: 1009.2448$].$

[25] D. Gorbunov and A. Tokareva, $R^{2}$-inflation with conformal SM Higgs field, arXiv: 1212.4466.

[26] F. Bezrukov and D. Gorbunov, Distinguishing between $R^{2}$-inflation and Higgs-inflation, Phys. Lett. B713 (2011) 365, [arXiv:1111.4397].

[27] G. F. Giudice and H. M. Lee, Unitarizing Higgs Inflation, Phys.Lett. B694 (2011) 294-300, [arXiv:1010.1417].

[28] S. Alekhin, J. Blumlein, and S. Moch, Parton distribution functions and benchmark cross sections at NNLO, arXiv:1202.2281. 